TRANSACTIONS OF THE

AMERICAN MATHEMATICAL SOCIETY

Volume 356, Number 10, Pages 4055-4073

S 0002-9947(04)03501-9

Article electronically published on February 27, 2004

\title{
CONSERVATION LAWS FOR A CLASS OF THIRD ORDER EVOLUTIONARY DIFFERENTIAL SYSTEMS
}

\author{
SUNG HO WANG
}

\begin{abstract}
Conservation laws of third order quasi-linear scalar evolution equations are studied via exterior differential system and characteristic cohomology. We find a subspace of 2 -forms in the infinite prolongation space in which every conservation law has a unique representative. Analysis of the structure of this subspace based upon the symbol of the differential equation leads to a universal integrability condition for an evolution equation to admit any higher order (weight) conservation laws. As an example, we give a complete classification of a class of evolution equations which admit conservation laws of the first three consecutive weights $-1,1,3$. The differential system describing the flow of a curve in the plane by the derivative of its curvature with respect to the arc length is also shown to exhibit the $K d V$ property, i.e., an infinite sequence of conservation laws of distinct weights.
\end{abstract}

\section{INTRODUCTION}

For a second order scalar evolutionary differential system (equation), it is known that every conservation law can be defined on the second jet space [BG2]. In fact, there is an upper bound on the order of the conservation laws for the even order scalar evolution equations $[\mathrm{F}]$. However, it is not true in the case of odd order equations, as is illustrated by the $K d V$ equation, and the infinite prolongation becomes necessary.

The lowest order conservation laws of the general quasi-linear third order scalar evolution equations are considered in $[\mathrm{F}]$, where the normal forms for the equations admitting such conservation laws are found. $[\underline{\mathrm{K}}]$ considers the evolution equations with higher order conservation laws, and $[\mathrm{M}]$ provides an extensive list of the equations that possess some kind of formal symmetry.

The purpose of this paper is to study the conservation laws of the third order scalar evolution equations of the form

$$
u_{t}=f\left(x, u, u_{x}\right) u_{x x x}+g\left(x, u, u_{x}, u_{x x}\right)
$$

via exterior differential systems and characteristic cohomology. The differential system and characteristic cohomology approach not only facilitates the computations involved, but it naturally gives rise to the notion of weight of a conservation law, which plays the role of the order of a conservation law in our treatment.

Received by the editors June 9, 2003 and, in revised form, July 8, 2003.

2000 Mathematics Subject Classification. Primary 35K25; Secondary 58A15.

Key words and phrases. Third order scalar evolution equation, exterior differential system, conservation law, characteristic cohomology. 
We find a universal integrability condition for equation (1) to admit a higher weight conservation law, which is expressed in terms of a curvature of a frame bundle associated to the equation. For instance, if this curvature does not vanish, every conservation law can be defined on the second jet space of solutions. Explicit computation of examples suggests the following conjecture:

Suppose a differential equation (1) has three conservation laws of distinct weights greater than or equal to 1 . Then it has an infinite sequence of conservation laws of distinct weights.

In Section 1, we solve the problem of equivalence, up to point transformations, for the differential systems that locally correspond to (1). Equivalence problem is solved on a principal bundle with certain two-dimensional group G as fibers, and we find a complete set of invariants whose functional relations determine the differential system up to the admissible transformations. The structure equations on the infinite prolongation space developed in Section 2 enable us to find a certain subspace, rather than a quotient space, in which every conservation law has a unique representative. We compute a rough normal form of a conservation law, and this leads to the universal integrability condition to admit any higher order conservation laws. The representation of the structure group $\mathrm{G}$ on the conservation laws gives the aforementioned notion of weight. In the final section, we compute the conservation laws of two classes of differential systems using the general theory. In particular, the $k_{1}$ flow, the flow of the curve in the plane by the derivative of its curvature with respect to arc length, is shown to exhibit the $K d V$ property, i.e., an infinite sequence of conservation laws of distinct weights.

In this paper, we only consider scalar conservation law, which is a special case of a Lie algebra $\mathfrak{g}$-valued conservation law. It would be interesting to study the case $\mathfrak{g}=s l(2, \mathrm{R})$ or $\mathfrak{g}=s u(2)$ for equation (1). Geometrically, a $\mathfrak{g}$-valued conservation law for (1) induces a map from (simply connected) solution surfaces to a Lie group with $\mathfrak{g}$ as its Lie algebra.

We thank Professor Robert Bryant for his guidance and support throughout this work.

\section{Structure equations on $\mathrm{F} \rightarrow \mathrm{M}$}

Consider the following 1-forms on $(t, x, u, p, q)$ space, where $p$ and $q$ stand for $u_{x}$ and $u_{x x}$ respectively, and $f(x, u, p) \neq 0$ :

$$
\begin{aligned}
w^{2} & =f(x, u, p) d t, \\
\eta_{0} & =f(x, u, p)(d u-p d x), \\
w^{1} & =d x, \\
\eta_{1} & =f(x, u, p)(d p-q d x), \\
\gamma_{2} & =f(x, u, p) d q+g(x, u, p, q) d x .
\end{aligned}
$$

Then the integral manifolds of the differential ideal

$$
\mathcal{I}=\left\{\eta_{0} \wedge w^{1}+\gamma_{2} \wedge w^{2}, \eta_{1} \wedge w^{2}, \eta_{0} \wedge w^{2}\right\} \cup\left\{\eta_{1} \wedge \eta_{0}\right\}
$$


on which $w^{1} \wedge w^{2} \neq 0$ correspond to the solutions of the pde (1). Note the 1 -forms introduced above satisfy the following structure equations:

$$
\begin{aligned}
d w^{2} & \equiv 0 \quad \bmod & w^{2}, & \\
d w^{1} & \equiv 0 \quad \bmod & w^{1}, \eta_{0}, & \\
d \eta_{0} & \equiv-\eta_{1} \wedge w^{1} & & \bmod \quad \eta_{0}, \\
d \eta_{1} & \equiv-\gamma_{2} \wedge w^{1} & & \bmod \quad \eta_{0}, \eta_{1},
\end{aligned}
$$

and if we put $T=\frac{\partial}{\partial t}$,

$$
\begin{aligned}
T\lrcorner w^{2} & \neq 0, \\
T\lrcorner \eta_{0} & \left.\left.=T\lrcorner w^{1}=T\right\lrcorner \eta_{1}=T\right\lrcorner \gamma_{2}=0, \\
T\lrcorner d \eta_{0} & \left.\left.=T\lrcorner d w^{1}=T\right\lrcorner d \eta_{1}=T\right\lrcorner d \gamma_{2}=0 .
\end{aligned}
$$

Conversely, if a coframe $\left\{w^{2}, \eta_{0}, w^{1}, \eta_{1}, \gamma_{2}\right\}$ of a five manifold $\mathrm{M}$ satisfies equations (3) and (4) for a nonzero vector field $T$, then the differential system (2) with independence condition $w^{1} \wedge w^{2} \neq 0$ corresponds (locally) to the pde

$$
u_{t}=f(x, u, p, q) u_{x x x}+g(x, u, p, q) \text {, }
$$

which includes (1) as a special case. We call such differential systems quasi-linear time-independent third order evolutionary differential systems.

Given such a differential system $\mathcal{I}$ on $\mathrm{M}, \mathrm{M}$ has a $G_{0} \subset \mathrm{Gl}(5, \mathrm{R})$ structure, where $G_{0}$ is a subgroup whose induced action on $\bigwedge^{2} T^{*} \mathrm{M}$ preserves the subspace defined by $\mathcal{I}$. Equivalently, the principal right $\mathrm{Gl}(5, \mathrm{R})$ bundle on $\mathrm{M}$ can be reduced to a $G_{0}$ bundle via $\mathcal{I}$. Let $F_{0}$ denote the $G_{0}$ bundle. A direct computation shows the elements of $G_{0}$ act on $\left\{w^{2}, \eta_{0}, w^{1}, \eta_{1}, \gamma_{2}\right\}$ by

$$
\left(\begin{array}{ccccc}
b^{3} & \cdot & \cdot & \cdot & \cdot \\
\cdot & a & \cdot & \cdot & \cdot \\
\cdot & e & b & \cdot & \cdot \\
\cdot & h & \cdot & \frac{a}{b} & \cdot \\
\cdot & l & \cdot & m & \frac{a}{b^{2}}
\end{array}\right)\left(\begin{array}{c}
w^{2} \\
\eta_{0} \\
w^{1} \\
\eta_{1} \\
\gamma_{2}
\end{array}\right)
$$

where '? denotes 0 . Note that if we allow the equivalence up to full contact transformations, $\eta_{1}$ term can be added to $w^{1}$. Thus on $F_{0}$, we have the following structure equations:

$$
\begin{aligned}
d\left(\begin{array}{c}
\omega^{2} \\
\theta_{0} \\
\omega^{1} \\
\theta_{1} \\
s_{2}
\end{array}\right)= & -\left(\begin{array}{ccccc}
3 \beta & \cdot & \cdot & \cdot & \cdot \\
\cdot & \alpha & \cdot & \cdot & \cdot \\
\cdot & \phi & \beta & \cdot & \cdot \\
\cdot & \phi_{1} & \cdot & \alpha-\beta & \cdot \\
\cdot & \psi & \cdot & \psi_{1} & \alpha-2 \beta
\end{array}\right) \wedge\left(\begin{array}{c}
\omega^{2} \\
\theta_{0} \\
\omega^{1} \\
\theta_{1} \\
s_{2}
\end{array}\right) \\
& +\left(\begin{array}{c}
-\theta_{1} \wedge \omega^{1}+\theta_{0} \wedge \epsilon_{0} \\
\Gamma^{1} \\
-s_{2} \wedge \omega^{1}+\theta_{0} \wedge \epsilon_{1}+\theta_{1} \wedge \epsilon_{2} \\
\Gamma_{2}
\end{array}\right),
\end{aligned}
$$


with

$$
\begin{aligned}
& d \beta=0, \\
& d \alpha \equiv-\phi_{1} \wedge \omega^{1}+\phi \wedge \theta_{1} \quad \bmod \theta_{0} .
\end{aligned}
$$

Here $\omega^{2}, \theta_{0}, \omega^{1}, \theta_{1}, s_{2}$ are tautological 1 forms, $\alpha, \beta, \phi, \phi_{1}, \psi, \psi_{1}$ are pseudoconnection forms, and

$$
\begin{array}{ll}
\epsilon_{0}, \epsilon_{1}, \epsilon_{2} \equiv 0 & \bmod \theta_{0}, \omega^{1}, \theta_{1}, s_{2}, \\
\Gamma^{1}, \Gamma_{2} & \text { quadratic in } \theta_{0}, \omega^{1}, \theta_{1}, s_{2},
\end{array}
$$

representing the torsion of the pseudo connection defined by $\alpha, \beta, \phi, \phi_{1}, \psi, \psi_{1}$.

This pseudo-connection is not uniquely defined, and modifying the pseudoconnection forms by the tautological 1 forms $\theta_{0}, \omega^{1}, \theta_{1}, s_{2}$, the torsion can be arranged as follows:

$$
\begin{aligned}
\epsilon_{0} & =\epsilon_{1}=0, \\
\Gamma^{1} & =a_{1} \theta_{1} \wedge \omega^{1}+R s_{2} \wedge \omega^{1}, \\
\epsilon_{2} & =a_{2} \omega^{1}+R s_{2}, \\
\Gamma_{2} & =a_{3} s_{2} \wedge \omega^{1},
\end{aligned}
$$

where $a_{1}, a_{2}, a_{3}, R$ are functions on $F_{0}$. Note that since the equivalence up to point transformations is allowed, the differential ideal generated by $\left\{\theta_{0}, \omega^{1}\right\}$ is integrable. The original differential system corresponds (locally) to an equation of the form

$$
u_{t}=f(x, u, p) u_{x x x}+g(x, u, p, q)
$$

iff $R=0$, which we assume from now on.

At this stage, no more absorption is possible without changing the form of the torsion given above. By taking the exterior derivative of the structure equations with the reduced torsion, we get

$$
\begin{aligned}
& d a_{1} \equiv \phi+a_{1}(\alpha-\beta) \quad \bmod \theta_{0}, \omega^{1}, \theta_{1}, \\
& d a_{2} \equiv 2 \phi_{1}-\psi_{1}+a_{2} \beta \quad \bmod \theta_{0}, \omega^{1}, \theta_{1}, s_{2}, \\
& d a_{3} \equiv \phi_{1}+\psi_{1}+a_{3} \beta, \quad \bmod \theta_{0}, \omega^{1}, \theta_{1}, s_{2} .
\end{aligned}
$$

Hence, the $G_{0}$ structure can be reduced to a $G_{1} \subset G_{0}$ structure by requiring $a_{1}=0, a_{2}=0, a_{3}=0$. In other words, we restrict to the sub-bundle $F_{1}$ of $F_{0}$ cut out by these relations. The structure equations on $F_{1}$ now become

$$
\begin{aligned}
d\left(\begin{array}{c}
\omega^{2} \\
\theta_{0} \\
\omega^{1} \\
\theta_{1} \\
s_{2}
\end{array}\right)= & \left(\begin{array}{ccccc}
3 \beta & \cdot & \cdot & \cdot & \cdot \\
\cdot & \alpha & \cdot & \cdot & \cdot \\
\cdot & \cdot & \beta & \cdot & \cdot \\
\cdot & \cdot & \cdot & \alpha-\beta & \cdot \\
\cdot & \psi & \cdot & \cdot & \alpha-2 \beta
\end{array}\right) \wedge\left(\begin{array}{c}
\omega^{2} \\
\theta_{0} \\
\omega^{1} \\
\theta_{1} \\
s_{2}
\end{array}\right) \\
& +\left(\begin{array}{c}
0 \\
-\theta_{1} \wedge \omega^{1} \\
\theta_{0} \wedge \phi \\
-s_{2} \wedge \omega^{1}+\theta_{0} \wedge \phi_{1} \\
\theta_{1} \wedge \psi_{1}
\end{array}\right)
\end{aligned}
$$


where $\phi \equiv 0 \bmod \theta_{0}, \omega^{1}, \theta_{1}$ and $\phi_{1}, \psi_{1} \equiv 0 \bmod \theta_{0}, \omega^{1}, \theta_{1}, s_{2}$. By modifying $\alpha, \psi$, we can arrange so that

$$
\begin{aligned}
\phi & =E \omega^{1}+F \theta_{1}, \\
\phi_{1} & =a_{4} \omega^{1}+K s_{2}, \\
\psi_{1} & =G \omega^{1}+2 K s_{2},
\end{aligned}
$$

where $E, F, G, K, a_{4}$ are now functions on $F_{1}$ that represent the unabsorbable torsion. Taking the exterior derivative again of the structure equations with this modified torsion gives, among other things, that $a_{4}$ can be translated to 0 by the group action corresponding to $\psi$. Thus we can reduce the $G_{1}$ structure $F_{1}$ to a twodimensional $\mathrm{G} \subset G_{1}$ structure $\mathrm{F}$ on which $a_{4}=0$, and $\psi \equiv 0 \bmod \theta_{0}, \omega^{1}, \theta_{1}, s_{2}$. The elements of $\mathrm{G}$ are of the form

$$
\left(\begin{array}{ccccc}
b^{3} & \cdot & \cdot & \cdot & \cdot \\
\cdot & a & \cdot & \cdot & \cdot \\
\cdot & \cdot & b & \cdot & \cdot \\
\cdot & \cdot & \cdot & \frac{a}{b} & \cdot \\
\cdot & \cdot & \cdot & \cdot & \frac{a}{b^{2}}
\end{array}\right)
$$

Summarizing the results, we have the following proposition.

Proposition 1.1. Let $\mathrm{M}$ be a five manifold with a quasi-linear time-independent third order evolutionary differential system $\mathcal{I}$ that locally corresponds to the equation of the form

$$
u_{t}=f(x, u, p) u_{x x x}+g(x, u, p, q) .
$$

Then there is an induced $\mathrm{G} \subset \mathrm{Gl}(5, \mathrm{R})$ bundle $\mathrm{F} \rightarrow \mathrm{M}$ with the structure equations

$$
\begin{aligned}
d\left(\begin{array}{c}
\omega^{2} \\
\theta_{0} \\
\omega^{1} \\
\theta_{1} \\
s_{2}
\end{array}\right) & =-\left(\begin{array}{ccccc}
3 \beta & \cdot & \cdot & \cdot & \cdot \\
\cdot & \alpha & \cdot & \cdot & \cdot \\
\cdot & \cdot & \beta & \cdot & \cdot \\
\cdot & \cdot & \cdot & \alpha-\beta & \cdot \\
\cdot & \cdot & \cdot & \cdot & \alpha-2 \beta
\end{array}\right) \wedge\left(\begin{array}{c}
\omega^{2} \\
\theta_{0} \\
\omega^{1} \\
\theta_{1} \\
s_{2}
\end{array}\right) \\
& +\left(\begin{array}{c}
0 \\
\theta_{0} \wedge\left(E \omega^{1}+F \theta_{1}\right) \\
-s_{2} \wedge \omega^{1}+K \theta_{0} \wedge s_{2} \\
\theta_{1} \wedge\left(G \omega^{1}+2 K s_{2}\right)+\theta_{0} \wedge\left(L \omega^{1}+M \theta_{1}+N s_{2}\right)
\end{array}\right), \\
d \beta=0, & 0 \\
d \alpha & =-E \theta_{1} \wedge \omega^{1}-K s_{2} \wedge \omega^{1}+\theta_{0} \wedge \epsilon,
\end{aligned}
$$

where $\epsilon \equiv 0 \bmod \omega^{1}, \theta_{1}, s_{2}$. The complete set of invariants of this $\mathrm{G}$ structure consists of $\epsilon, E, F, G, K, L, M, N$ and their successive covariant derivatives. If all of the invariants are 0 , the differential system is equivalent to the system generated by $u_{t}=u_{x x x}$ via a point transformation, which preserves the ideals generated by $\left\{\omega^{2}\right\}$ and $\left\{\theta_{0}, \omega^{1}\right\}$. By construction, if $\mathcal{I}$ and $\mathcal{I}^{\prime}$ are two differential systems such that $\varphi^{*} \mathcal{I}^{\prime}=\mathcal{I}$ for a point transformation $\varphi$ of $\mathrm{M}$, then the induced $\mathrm{G}$ structures $\mathrm{F}$ and $\mathrm{F}^{\prime}$ are equivalent.

Given an evolutionary pde (1), we mention that $K=0$ iff the pde is of the form

$$
u_{t}=(f(x, u, p) q)_{x}+g(x, u, p) q+h(x, u, p) .
$$


In this case, $E=-N$, and $N=0$ iff

$$
\begin{aligned}
4 f_{p}^{2} & =3 f f_{p p}, \\
4\left(f_{p} f_{x}+p f_{p} f_{u}\right) & =3 f\left(f_{p x}+p f_{p u}-f_{u}\right) .
\end{aligned}
$$

2. Structure equations on $\mathrm{F}_{\infty} \rightarrow \mathrm{F} \rightarrow \mathrm{M}$

Consider the linear pde

$$
u_{t}=u_{x x x}
$$

Since every $t$ derivative of $u$ can be replaced by $x x x$ derivative for any solution $u$, we may define the $(k+3)$ rd jet space $J_{k+3}$ of $u$ as the space with coordinates

$$
\left\{x, t, u=p_{0}, p_{1}, p_{2}, p_{3}, \ldots, p_{k+3}\right\},
$$

where $p_{1}=u_{x}, p_{2}=u_{x x}$, and $p_{i}=u_{x x x \ldots x}$. In terms of differential forms, we introduce the following 1 -forms on $J_{k+3}$.

$$
\begin{aligned}
& w^{1}=d x, w^{2}=d t, \\
& \gamma_{i}=d p_{i} \quad \text { for } 0 \leq i \leq k+3 \text {, } \\
& \eta_{i}=\gamma_{i}-p_{i+1} d x \quad \text { for } 0 \leq i \leq k+2 \text {, } \\
& \phi_{i}=\eta_{i} \quad-p_{i+3} d t \quad \text { for } 0 \leq i \leq k \text {. }
\end{aligned}
$$

Then $\left\{w^{1}, w^{2}, \phi_{0}, \phi_{1}, \ldots, \phi_{k}, \eta_{k+1}, \eta_{k+2}, \gamma_{k+3}\right\}$ is a basis of $T^{*} J_{k+3}$, and they satisfy the following structure equations:

$$
\begin{aligned}
-d \eta_{i} & =\eta_{i+1} \wedge w^{1}=\gamma_{i+1} \wedge w^{1} \quad \text { for } 0 \leq i \leq k+1, \\
-d \eta_{k+2} & =\gamma_{k+3} \wedge w^{1} \\
-d \phi_{i} & =\phi_{i+1} \wedge w^{1}+\phi_{i+3} \wedge w^{2}, \\
& =\eta_{i+1} \wedge w^{1}+\gamma_{i+3} \wedge w^{2} \quad \text { for } 0 \leq i \leq k-3, \\
-d \phi_{k-2} & =\phi_{k-1} \wedge w^{1}+\eta_{k+1} \wedge w^{2} \\
-d \phi_{k-1} & =\phi_{k} \wedge w^{1}+\eta_{k+2} \wedge w^{2} \\
-d \phi_{k} & =\eta_{k+1} \wedge w^{1}+\gamma_{k+3} \wedge w^{2} .
\end{aligned}
$$

The original differential system for $u_{t}=u_{x x x}$ on $\left(x, t, u, p_{1}, p_{2}\right)$ space is generated by

$$
\begin{aligned}
& \left\{\eta_{0} \wedge w^{1}+\gamma_{2} \wedge w^{2}\right\} \\
\cup & \left\{\eta_{1} \wedge w^{2}, \eta_{0} \wedge w^{2}\right\} \\
\cup & \left\{\eta_{1} \wedge \eta_{0}\right\},
\end{aligned}
$$

and the structure equations show the $(k+3)$ rd prolongation of this differential system on $J_{k+3}$ is generated by

$$
\begin{aligned}
& \left\{\phi_{0}, \phi_{1}, \phi_{2}, \ldots, \phi_{k}\right\} \\
\cup & \left\{\eta_{k+1} \wedge w^{1}+\gamma_{k+3} \wedge w^{2}\right\} \\
\cup & \left\{\eta_{k+2} \wedge w^{2}, \eta_{k+1} \wedge w^{2}\right\} \\
\cup & \left\{\eta_{k+2} \wedge \eta_{k+1}\right\} .
\end{aligned}
$$

For a general quasi-linear evolutionary differential system $\mathcal{I}$ on a five manifold $\mathrm{M}$, let $\mathrm{M}_{k+3}$ denote the $(k+3)$ rd prolongation space, $\mathrm{M}_{\infty}=\lim _{k \rightarrow \infty} \mathrm{M}_{k+3}$, and 
let $p_{3}, p_{4}, p_{5}, \ldots$ be the prolongation variables. Also define $\mathrm{F}_{k+3}$ and $\mathrm{F}_{\infty}$ as the pullback of $\mathrm{M}_{k+3}$ and $\mathrm{M}_{\infty}$ via $\pi$ respectively, where $\pi$ is the projection map $\pi$ : $\mathrm{F} \rightarrow \mathrm{M}$ of the $\mathrm{G}$ structure defined earlier. Motivated by the linear example above, we observe the following.

Theorem 2.1. There is a sequence of time-independent functions $r_{k+3}$ on $\mathrm{F}_{k+2}$ for $k \geq 2$ such that if we define

$$
\begin{array}{rlrl}
s_{i} & =d p_{i}+p_{i}(\alpha-i \beta) & \text { for } i \geq 3, \\
\sigma_{2} & =s_{2}, \quad \sigma_{3}=s_{3}, \quad \sigma_{4}=s_{4}+p_{3} K s_{2}, \\
\sigma_{k+3} & =s_{k+3}+r_{k+3} \omega^{1} & \text { for } k \geq 2, \\
\theta_{k} & =\sigma_{k}-p_{k+1} w^{1} & \text { for } k \geq 2, \\
\pi_{k} & =\theta_{k} \quad-p_{k+3} \omega^{2} & \text { for } k \geq 0,
\end{array}
$$

then

1. $\left\{\alpha, \beta, \omega^{1}, \omega^{2}, \pi_{0}, \pi_{1}, \ldots, \pi_{k}, \theta_{k+1}, \theta_{k+2}, \sigma_{k+3}\right\}$ is a basis of $T^{*} \mathrm{~F}_{k+3}$ for $k \geq 0$.

2. For $k \geq 0$,

$$
\begin{aligned}
-d \pi_{i} & \equiv \pi_{i+1} \wedge \omega^{1}+\pi_{i+3} \wedge \omega^{2} \quad \bmod \pi_{0}, \ldots, \pi_{i} \quad \text { for } 0 \leq i \leq k-3, \\
-d \pi_{k-2} & \equiv \pi_{k-1} \wedge \omega^{1}+\theta_{k+1} \wedge \omega^{2} \quad \bmod \pi_{0}, \ldots, \pi_{k-2}, \\
-d \pi_{k-1} & \equiv \pi_{k} \wedge \omega^{1}+\theta_{k+2} \wedge \omega^{2} \quad \bmod \pi_{0}, \ldots, \pi_{k-1}, \\
-d \pi_{k} & \equiv \theta_{k+1} \wedge \omega^{1}+\sigma_{k+3} \wedge \omega^{2} \quad \bmod \pi_{0}, \ldots, \pi_{k} .
\end{aligned}
$$

3. For $i \geq 0$,

$$
-d \theta_{i} \equiv(\alpha-i \beta) \wedge \theta_{i}+\theta_{i+1} \wedge \omega^{1} \quad \bmod \quad \theta_{0}, \theta_{1}, \ldots, \theta_{i-2}, \theta_{i-1} .
$$

Note that 2. implies the $(k+3)$ rd prolonged ideal $\mathcal{I}_{k+3}$ on $\mathrm{F}_{k+3}$ is generated by

$$
\begin{aligned}
\mathcal{I}_{k+3}= & \left\{\pi_{0}, \pi_{1}, \pi_{2}, \ldots, \pi_{k}\right\} \\
& \cup\left\{\theta_{k+1} \wedge \omega^{1}+\sigma_{k+3} \wedge \omega^{2}\right\} \\
& \cup\left\{\theta_{k+2} \wedge \omega^{2}, \theta_{k+1} \wedge \omega^{2}\right\} \\
& \cup\left\{\theta_{k+2} \wedge \theta_{k+1}\right\} .
\end{aligned}
$$

Also by definition,

$$
\begin{aligned}
\pi_{i} \wedge \omega^{1}+\theta_{i+2} \wedge \omega^{2} & =\theta_{i} \wedge \omega^{1}+\sigma_{i+2} \wedge \omega^{2}, \\
\theta_{i} \wedge \omega^{1} & =\sigma_{i} \wedge \omega^{1} \\
\pi_{i} \wedge \omega^{2} & =\theta_{i} \wedge \omega^{2} .
\end{aligned}
$$

Proof. 1. It follows from the definition, as long as $r_{k+3} \in C^{\infty}\left(\mathrm{F}_{k+2}\right)$, which is checked below.

2. First, we consider the original differential system $\mathcal{I}=\mathcal{I}_{2}$ on $\mathrm{F}=\mathrm{F}_{2}$. (For simplicity, we use the same letter $\mathcal{I}$ to denote $\pi^{*} \mathcal{I}$ on $\mathrm{F}$, where $\pi: \mathrm{F} \rightarrow \mathrm{M}$ )

$$
\begin{aligned}
\mathcal{I}=\mathcal{I}_{2} & =\left\{\theta_{0} \wedge \omega^{1}+\sigma_{2} \wedge \omega^{2}\right\}, \\
& \cup\left\{\theta_{1} \wedge \omega^{2}, \theta_{0} \wedge \omega^{2}\right\} \\
& \cup\left\{\theta_{1} \wedge \theta_{0}\right\} .
\end{aligned}
$$


Since $\theta_{0} \wedge \omega^{2} \in \mathcal{I}, \theta_{0}$ is a multiple of $\omega^{2}$ on every integral manifold of $\mathcal{I}$ on which $\omega^{1} \wedge \omega^{2} \neq 0$. This suggests introducing

$$
\begin{aligned}
\mathrm{F}_{3} & =\mathrm{F} \times\left\{p_{3}\right\}, \\
\pi_{0} & =\theta_{0}-p_{3} \omega^{2} .
\end{aligned}
$$

But,

$$
\theta_{0} \wedge \omega^{1}+s_{2} \wedge \omega^{2} \equiv \theta_{2} \wedge \omega^{2} \quad \bmod \pi_{0}
$$

by definition of $\theta_{2}$. Also from the structure equations on $\mathrm{F}$,

$$
-d \pi_{0}=-d\left(\theta_{0}-p_{3} \omega^{2}\right) \equiv \theta_{1} \wedge \omega^{1}+\sigma_{3} \wedge \omega^{2} \quad \bmod \pi_{0} .
$$

This implies $\mathcal{I}_{3}$ is generated by

$$
\begin{aligned}
\mathcal{I}_{3}= & \left\{\pi_{0}\right\} \\
& \cup\left\{\theta_{1} \wedge \omega^{1}+\sigma_{3} \wedge \omega^{2}\right\} \\
& \cup\left\{\theta_{2} \wedge \omega^{2}, \theta_{1} \wedge \omega^{2}\right\} \\
& \cup\left\{\theta_{2} \wedge \theta_{1}\right\},
\end{aligned}
$$

which is the $p_{3}$ prolongation $\mathcal{I}_{3}$ of $\mathcal{I}$. From the arguments above, it is clear that every integral manifold of $\mathcal{I}_{3}$ in $\mathrm{F}_{3}$ on which $\omega^{1} \wedge \omega^{2} \neq 0$ is a graph over an integral manifold of $\mathcal{I}$ in F. Note that

$$
\left\{\alpha, \beta, \omega^{1}, \omega^{2}, \pi_{0}, \theta_{1}, \theta_{2}, \sigma_{3}\right\}
$$

is a basis of $T^{*} \mathrm{~F}_{3}$.

Next, we define the $p_{4}$ prolongation by a similar procedure, introducing

$$
\begin{aligned}
\mathrm{F}_{4} & =\mathrm{F}_{3} \times\left\{p_{4}\right\}, \\
\pi_{1} & =\theta_{1}-p_{4} \omega^{2} .
\end{aligned}
$$

Then,

$$
\theta_{1} \wedge \omega^{1}+\sigma_{3} \wedge \omega^{2} \equiv \theta_{3} \wedge \omega^{2} \quad \bmod \pi_{0}, \pi_{1}
$$

and

$$
-d \pi_{1}=-d\left(\theta_{1}-p_{4} \omega^{2}\right) \equiv \theta_{2} \wedge \omega^{1}+\sigma_{4} \wedge \omega^{2} \quad \bmod \pi_{0}, \pi_{1} .
$$

Hence,

$$
\begin{aligned}
\mathcal{I}_{4}= & \left\{\pi_{0}, \pi_{1}\right\} \\
& \cup\left\{\theta_{2} \wedge \omega^{1}+\sigma_{4} \wedge \omega^{2}\right\} \\
& \cup\left\{\theta_{3} \wedge \omega^{2}, \theta_{2} \wedge \omega^{2}\right\} \\
& \cup\left\{\theta_{3} \wedge \theta_{2}\right\} .
\end{aligned}
$$

The claim for $p_{5}, p_{6}, p_{7}$ prolongations can also be checked as above by direct computations.

Inductively, suppose there exists a sequence of time independent functions $r_{5}$, $r_{6}, \ldots, r_{k+3}, k \geq 4$, such that the structure equations in 2 . of the theorem is true. It suffices to show there exists time independent $r_{k+4} \in C^{\infty}\left(\mathrm{F}_{k+3}\right)$ such that

$$
-d \pi_{k+1} \equiv \theta_{k+2} \wedge \omega^{1}+\sigma_{k+4} \wedge \omega^{2} \bmod \pi_{0}, \ldots, \pi_{k+1} .
$$

We only verify the claim $\bmod \alpha, \beta$ for simplicity. The terms containing $\alpha$ and $\beta$ can be checked by counting the weights of the representation of the structural group $\mathrm{G}$ on $\mathrm{F}_{\infty}$. For brevity, we use $\left\{p_{i}\right\}$ to denote a time independent function on $\mathrm{F}_{i}$, 
i.e., it's an algebraic function of $p_{3}, p_{4}, \ldots, p_{i}$, and the complete set of invariants of the original $\mathrm{G}$ structure $\mathrm{F} \rightarrow \mathrm{M}$.

Notice from the structure equation on $\mathrm{F} \rightarrow \mathrm{M}$,

$$
\begin{aligned}
d \omega^{2} & \equiv 0, \\
d \theta_{0} & \equiv p_{4} \omega^{1} \wedge \omega^{2}, \\
d \omega^{1} & \equiv\left\{p_{3}\right\} \omega^{1} \wedge \omega^{2}, \\
d \theta_{1} & \equiv\left\{p_{5}\right\} \omega^{1} \wedge \omega^{2}, \\
d s_{2} & \equiv\left\{p_{4}\right\} \omega^{1} \wedge \omega^{2}, \\
d \alpha & \equiv\left\{p_{5}\right\} \omega^{1} \wedge \omega^{2} \quad \bmod \alpha, \beta, \pi_{0}, \pi_{1}, \pi_{2} .
\end{aligned}
$$

Thus,

$$
\begin{aligned}
d \pi_{k+1}= & d\left(\theta_{k+1}-p_{k+4} \omega^{2}\right) \\
= & d\left(d p_{k+1}+p_{k+1}(\alpha-(k+1) \beta)+r_{k+1} \omega^{1}-p_{k+2} \omega^{1}-p_{k+4} \omega^{2}\right) \\
\equiv & p_{k+1}\left\{p_{5}\right\} \omega^{1} \wedge \omega^{2}+d r_{k+1} \wedge \omega^{1}+r_{k+1}\left\{p_{3}\right\} \omega^{1} \wedge \omega^{2} \\
& +p_{k+2}\left\{p_{3}\right\} \omega^{1} \wedge \omega^{2}-d p_{k+2} \wedge \omega^{1}-d p_{k+4} \wedge \omega^{2} \\
\equiv & -d p_{k+2} \wedge \omega^{1}-d p_{k+4} \wedge \omega^{2}+d r_{k+1} \wedge \omega^{1}+\left\{p_{k+3}\right\} \omega^{1} \wedge \omega^{2} \\
& \bmod \alpha, \beta, \pi_{0}, \pi_{1}, \pi_{2}
\end{aligned}
$$

since $r_{k+1} \in C^{\infty}\left(\mathrm{F}_{k}\right)$ is time independent by induction hypothesis and $k \geq 4$. Also,

$$
\theta_{k+2} \wedge \omega^{1}+\sigma_{k+4} \wedge \omega^{2} \equiv d p_{k+2} \wedge \omega^{1}+d p_{k+4} \wedge \omega^{2}+r_{k+4} \omega^{1} \wedge \omega^{2} \quad \bmod \alpha, \beta,
$$

by definition of $r_{k+4}$. Now, it is sufficient to show

$$
d r_{k+1} \wedge \omega^{1} \equiv\left\{p_{k+3}\right\} \omega^{1} \wedge \omega^{2} \bmod \alpha, \beta, \pi_{0}, \ldots, \pi_{k} .
$$

But, again, $r_{k+1} \in C^{\infty}\left(\mathrm{F}_{k}\right)$ is time independent and by definition of the 1-form $\pi_{i}$,

$$
d p_{i} \equiv p_{i+3} \omega^{2} \quad \bmod \alpha, \beta, \omega^{1}, \pi_{i} .
$$

In particular,

$$
d p_{k} \equiv p_{k+3} \omega^{2} \quad \bmod \alpha, \beta, \omega^{1}, \pi_{k},
$$

and $r_{k+4} \in C^{\infty}\left(\mathrm{F}_{k+3}\right)$ is uniquely determined.

3. Straightforward computations. We omit the proof.

\section{Conservation laws}

In this section, we compute a rough normal form of conservation laws using the structure equations on $\mathrm{F}_{\infty} \rightarrow \mathrm{F}$. This leads to a universal integrability condition to admit any higher weight (to be defined in 3.2) conservation laws in terms of vanishing of certain invariants of the bundle $\mathrm{F} \rightarrow \mathrm{M}$ defined in Section 1. Throughout this section, we denote by $\bigwedge^{p} \mathcal{I}_{k+3}$ the space of $p$-forms on $\mathrm{F}_{k+3}$ in the ideal $\mathcal{I}_{k+3}$.

3.1. $\wedge^{2} \mathcal{I}_{\infty} / d \mathcal{I}_{\infty}$. Following [BG1], we start with a definition.

Definition 3.1. A conservation law is a class $[\Phi] \in \bigwedge^{2} \mathcal{I}_{\infty} / d \mathcal{I}_{\infty}$ generated by a closed 2 -form $\Phi$. The space of conservation laws is denoted by $\mathcal{C}_{\infty}$. 
Here we say $d \mathcal{I}_{\infty}$ is the subspace of exact 2-forms in the ideal of the form $d\left(\lambda_{0} \pi_{0}+\lambda_{1} \pi_{1}+\cdots+\lambda_{m} \pi_{m}\right)$ for some functions $\lambda_{i}$ on $\mathrm{F}_{\infty}$. For such a closed 2 -form $\Phi$, consider a 1 -form $\theta$ such that

$$
d \theta=\Phi .
$$

If $N$ is an integral manifold of $\mathcal{I}$, hence of $\mathcal{I}_{\infty}$, it follows by Stokes theorem that

$$
0=\int_{N} \Phi=\int_{N} d \theta=\int_{\partial N} \theta
$$

which becomes a classical conservation law for an evolutionary pde once we impose nice decay conditions on the solutions. BG1 shows that, at least locally, the cohomology $\mathcal{C}_{\infty}$ defined as above is isomorphic to the classical space of conservation laws

$$
\mathcal{C}_{\infty} \cong\left\{\theta \in \bigwedge^{1} \mathrm{~F}_{\infty} \mid d \theta \equiv 0 \quad \bmod \mathcal{I}_{\infty}\right\} /\left\{\left\{\bigwedge^{1} \mathcal{I}_{\infty}\right\} \cup\left\{d f \mid f \in C^{\infty}\left(\mathrm{F}_{\infty}\right)\right\}\right\} .
$$

Instead of dealing directly with the quotient $\bigwedge^{2} \mathcal{I}_{\infty} / d \mathcal{I}_{\infty}$, we introduce a section of the projection

$$
\bigwedge^{2} \mathcal{I}_{\infty} \rightarrow \bigwedge^{2} \mathcal{I}_{\infty} / d \mathcal{I}_{\infty}
$$

Theorem 3.2. There exists a sequence of subspaces $\mathcal{H}_{k+3} \subset \bigwedge^{2} \mathcal{I}_{k+3}$ for $k \geq 0$ such that

1. $\mathcal{H}_{k+3}=\mathcal{H}_{k+2} \oplus\left\{\theta_{k+2} \wedge \theta_{k+1}\right\} \oplus\left\{\theta_{k+2} \wedge \theta_{k}\right\}$

$$
\oplus\left\{\sigma_{k+3} \wedge \pi_{i} \mid 0 \leq i \leq k\right\}
$$$$
\oplus\left\{\theta_{k+2} \wedge \omega^{2}\right\} \text {, and }
$$$$
\mathcal{H}_{k+3} \cong \bigwedge^{2} \mathcal{I}_{k+3} / d \mathcal{I}_{k+3} \text { for } k \geq 0 .
$$

2. Set $\mathcal{H}_{\infty}=\lim _{k \rightarrow \infty} \mathcal{H}_{k+3}$ then,

$$
\mathcal{H}_{\infty} \cong \bigwedge^{2} \mathcal{I}_{\infty} / d \mathcal{I}_{\infty}
$$

We define

$$
\begin{aligned}
\mathcal{H}_{1} & =\left\{\theta_{0} \wedge \omega^{1}+\sigma_{2} \wedge \omega^{2}\right\} \\
& \oplus\left\{\theta_{1} \wedge \omega^{2}, \theta_{0} \wedge \omega^{2}\right\}, \\
\mathcal{H}_{2} & =\mathcal{H}_{1} \oplus\left\{\theta_{1} \wedge \theta_{0}\right\} .
\end{aligned}
$$

In this theorem, the expression $\left\{\theta_{1} \wedge \theta_{0}\right\}$, for example, means the subspace generated by the 2 -form $\theta_{1} \wedge \theta_{0}$.

Corollary 3.3. Every conservation law has a unique representative in $\mathcal{H}_{\infty}$.

Proof of Theorem 3.2. 1. Suppose $\Phi \in \bigwedge^{2} \mathcal{I}_{k+3}$ for some $k \geq 0$. Then

$$
\begin{aligned}
\Phi & =\pi_{0} \wedge \epsilon_{0}+\pi_{1} \wedge \epsilon_{1}+\cdots+\pi_{k} \wedge \epsilon_{k}+\sum_{i, j=0}^{k} c_{i j} \pi_{i} \wedge \pi_{j} \\
& +v\left(\theta_{k+1} \wedge \omega^{1}+\sigma_{k+3} \wedge \omega^{2}\right) \\
& +y \theta_{k+2} \wedge \omega^{2}+z \theta_{k+1} \wedge \omega^{2} \\
& +w \theta_{k+2} \wedge \theta_{k+1}
\end{aligned}
$$


where $\epsilon_{i} \equiv 0 \bmod \omega^{1}, \omega^{2}, \theta_{k+1}, \theta_{k+2}, \sigma_{k+3}$ and $c_{i j}=-c_{j i}, v, y, z, w$ are functions on $\mathrm{F}_{k+3}$. Since

$$
-d \pi_{k} \equiv \theta_{k+1} \wedge \omega^{1}+\sigma_{k+3} \wedge \omega^{2} \bmod \pi_{0}, \pi_{1}, \ldots, \pi_{k},
$$

we may take $v=0$ by modifying $\epsilon_{i}$ and $c_{i j}$ if necessary mod $d \mathcal{I}_{k+3}$. Also, since

$$
-\pi_{i} \wedge \omega^{1} \equiv \theta_{i+2} \wedge \omega^{2} \quad \bmod d \pi_{i-1}, \pi_{i-1}, \ldots, \pi_{0} \quad \text { for } 1 \leq i \leq k,
$$

we may take $\epsilon_{i} \equiv 0 \bmod \omega^{2}, \theta_{k+1}, \theta_{k+2}, \sigma_{k+3}$ for $1 \leq i \leq k$ by successively modifying $\epsilon_{i}$ and $c_{i j}$ if necessary. At this stage, no more modification $\bmod d \mathcal{I}_{k+3}$ is possible. We denote this subspace by $H_{k+3} \subset \bigwedge^{2} \mathcal{I}_{k+3}$ for each $k \geq 0$, which satisfies

$$
H_{k+3} \oplus d \mathcal{I}_{k+3}=\bigwedge^{2} \mathcal{I}_{k+3} .
$$

Next, we modify $H_{k+3}$ by $d \mathcal{I}_{k+3}$ to construct another subspace $\mathcal{H}_{k+3} \subset \bigwedge^{2} \mathcal{I}_{k+3}$ complementary to $d \mathcal{I}_{k+3}$, but which satisfies

$$
\mathcal{H}_{k+3} \subset \mathcal{H}_{k+4} \text {. }
$$

First, note that as we prolong from $\mathcal{I}_{k+3}$ to $\mathcal{I}_{k+4}$,

$$
\begin{aligned}
\pi_{i} & \rightarrow \pi_{i} \quad \text { for } 0 \leq i \leq k, \\
\theta_{k+1} \rightarrow & \pi_{k+1}, \\
\theta_{k+2} \rightarrow & \theta_{k+2}, \\
\sigma_{k+3} \rightarrow & \theta_{k+3}, \\
& \sigma_{k+4} .
\end{aligned}
$$

This observation together with the fact

$$
\begin{aligned}
\pi_{i} \wedge \pi_{k+1} & \equiv \pi_{i} \wedge \theta_{k+1} \quad \bmod \pi_{i} \wedge \omega^{2} \\
\pi_{i} \wedge \theta_{k+3} & \equiv \pi_{i} \wedge \sigma_{k+3} \quad \bmod \pi_{i} \wedge \omega^{1}, \\
-\pi_{i} \wedge \omega^{1} & \equiv \theta_{i+2} \wedge \omega^{2} \quad \bmod d \pi_{i-1}, \pi_{i-1}, \ldots, \pi_{0} \quad \text { for } 1 \leq i \leq k, \\
\theta_{k+3} \wedge \pi_{k+1} & \equiv \theta_{k+3} \wedge \theta_{k+1} \quad \bmod \theta_{k+3} \wedge \omega^{2}
\end{aligned}
$$

implies

$$
\begin{aligned}
H_{k+4} \equiv H_{k+3} & \oplus\left\{\theta_{k+3} \wedge \theta_{k+2}\right\} \oplus\left\{\theta_{k+3} \wedge \theta_{k+1}\right\} \\
& \oplus\left\{\sigma_{k+4} \wedge \pi_{i} \mid 0 \leq i \leq k+1\right\} \\
& \oplus\left\{\theta_{k+3} \wedge \omega^{2}\right\} \quad \bmod d \mathcal{I}_{k+4}
\end{aligned}
$$

We inductively define $\mathcal{H}_{k+4}$ by

$$
\begin{aligned}
\mathcal{H}_{k+4}=\mathcal{H}_{k+3} & \oplus\left\{\theta_{k+3} \wedge \theta_{k+2}\right\} \oplus\left\{\theta_{k+3} \wedge \theta_{k+1}\right\} \\
& \oplus\left\{\sigma_{k+4} \wedge \pi_{i} \mid 0 \leq i \leq k+1\right\} \\
& \oplus\left\{\theta_{k+3} \wedge \omega^{2}\right\} .
\end{aligned}
$$

2. Suppose

$$
d\left(\lambda_{0} \pi_{0}+\lambda_{1} \pi_{1}+\cdots+\lambda_{m} \pi_{m}\right) \in \mathcal{H}_{k+3} .
$$

There exists $l \geq k$ such that every $\lambda_{i} \in C^{\infty}\left(F_{l+3}\right)$ and

$$
\lambda_{0} \pi_{0}+\lambda_{1} \pi_{1}+\cdots+\lambda_{m} \pi_{m} \in \mathcal{I}_{l+3} .
$$

But $\mathcal{H}_{k+3} \subset \mathcal{H}_{l+3}, \mathcal{H}_{l+3} \cap d \mathcal{I}_{l+3}=0$ implies

$$
d\left(\lambda_{0} \pi_{0}+\lambda_{1} \pi_{1}+\cdots+\lambda_{m} \pi_{m}\right)=0 .
$$


3.2. Sequence of linear differential systems. Consider a generator $\Phi$ of a conservation law that belongs to $\mathcal{H}_{k+3}$ only pointwise:

$$
\begin{aligned}
\Phi & =A\left(\theta_{0} \wedge \omega^{1}+\sigma_{2} \wedge \omega^{2}\right)-B \theta_{1} \wedge \omega^{2}+C \theta_{0} \wedge \omega^{2} \\
& +\sum_{i=0}^{k+1} T_{i+1, i} \theta_{i+1} \wedge \theta_{i} \\
& +\sum_{i=0}^{k} X_{i+2, i} \theta_{i+2} \wedge \theta_{i}+\sum_{j=0}^{k} \sum_{i=0}^{j} X_{j+3, i} \sigma_{j+3} \wedge \pi_{i} \\
& +\sum_{i=2}^{k+2} Y_{i} \theta_{i} \wedge \omega^{2},
\end{aligned}
$$

where $A, B, C, T_{i+1, i}, X_{i+2, i}, X_{j+3, i}$, and $Y_{i}$ are functions on $\mathrm{F}_{\infty}$. We first note that all of the coefficients $A, B, C, T_{i+1, i}, X_{i+2, i}, X_{j+3, i}, Y_{i}$ must be functions on $\mathrm{F}_{k+3}$ in order for $\Phi$ to be closed, which in turn justifies the decomposition of $\bigwedge^{2} \mathcal{I}_{\infty}$ in Theorem 3.2. Moreover, the coefficient $\mathrm{T}_{k+2, k+1}$ of highest weight $2 k+3$ under the representation corresponding to the connection form $\beta$ of the original $\mathrm{G}$ structure $\mathrm{F} \rightarrow \mathrm{M}$ turns out to indicate exactly when $\Phi \in \mathcal{H}_{k+2} \subset \mathcal{H}_{k+3}$.

Throughout this section, $\Phi$ will always be assumed to be closed, and for the reasons to follow, we call $A, B, C$, and $\left\{T_{i+1, i}\right\}_{i=0}^{\infty}$ the principal coefficients.

Theorem 3.4. For $\Phi \in \mathcal{H}_{k+3}, k \geq-1$,

1. $\Phi \in \mathcal{H}_{k+2} \subset \mathcal{H}_{k+3} \quad$ iff $\quad T_{k+2, k+1}=0$.

2. $d T_{k+2, k+1} \equiv T_{k+2, k+1}\left(2 \alpha-(2 k+3) \beta+2 N \theta_{0}-2 K \theta_{1}\right) \bmod \omega^{2}$.

Corollary 3.5. If $d\left(\alpha+N \theta_{0}-K \theta_{1}\right) \neq 0$, every conservation law belongs to $\mathcal{H}_{1}$.

Corollary 3.5 follows from the fact the differential system is time independent and $d \beta=0$. In local coordinates, an evolution equation (1) satisfies

$$
d\left(\alpha+N \theta_{0}-K \theta_{1}\right)=0
$$

iff $g(x, u, p, q)$ is at most quadratic in $q$, and $f(x, u, p)$ and $g(x, u, p, q)$ satisfy some fifth order partial differential relations. We also mention that if $K$ is not 0 , there is no conservation laws in $\mathcal{H}_{1}$.

Proof of Theorem 3.4. We assume $k \geq 1$. The cases $k=-1,0$ can be checked by direct computations.

1. From the results in the preceding section, we need to show

$$
X_{k+2, k}=Y_{k+2}=X_{k+3, i}=0 \text { for } 0 \leq i \leq k
$$

assuming $T_{k+2, k+1}=0$. Rather than getting into the detail of the computation, we present a sketch of the arguments involved.

(a) $d \Phi \equiv 0 \bmod \omega^{2}, \pi_{0}, \ldots, \pi_{k}$ gives $X_{k+2, k}=X_{k+3, k}=0$.

(b) $d \Phi \equiv 0 \bmod \pi_{0}, \ldots, \pi_{k}, \theta_{k+1}, \theta_{k+2}$ gives $Y_{k+2}=0$. Here we note that $d \theta_{i} \equiv$ $d \pi_{i} \bmod \omega^{2}$.

(c) First,

$$
d \Phi \equiv 0 \quad \bmod \quad \omega^{2}, \theta_{0}, \ldots, \tilde{\theta_{k}}, \theta_{k+1}, \sigma_{k+2}
$$

gives $X_{k+3, k-1}=0$. Inductively,

$$
d \Phi \equiv 0 \quad \bmod \quad \omega^{2}, \theta_{0}, \ldots, \widetilde{\theta}_{i}, \theta_{i+1}, \theta_{i+2}, \sigma_{i+3}, \ldots, \sigma_{k+2}
$$


gives $X_{k+3, i-1}=0$ for $k-1 \geq i \geq 1$. Other than the structure equations in Theorem 2.1, we make use of the fact

$$
\begin{aligned}
d \sigma_{i} & \equiv d r_{i} \wedge \omega^{1} \quad \bmod \quad \alpha, \beta, \theta_{0}, \theta_{1}, \theta_{2} \\
& \equiv 0 \quad \bmod \quad \alpha, \beta, \theta_{0}, \ldots, \theta_{i-2}, \sigma_{i-1}
\end{aligned}
$$

for $r_{i}$ is a function on $\mathrm{F}_{i-1}$ that is time independent.

2. We only show

$$
d T_{k+2, k+1} \equiv 0 \quad \bmod \quad \alpha, \beta, \omega^{2}, \theta_{0}, \theta_{1} .
$$

The rest of the assertion follows easily from this. Put

$$
\begin{aligned}
d T_{k+2, k+1} \equiv & T_{k+2, k+1}^{-1} \omega^{1}+T_{k+2, k+1}^{0} \theta_{0}+\ldots+T_{k+2, k+1}^{k+2} \theta_{k+2}+T_{k+2, k+1}^{k+3} \sigma_{k+3} \\
& \bmod \alpha, \beta, \omega^{2}
\end{aligned}
$$

to express the covariant derivative of $T_{k+2, k+1}$.

First, $d \Phi \equiv 0 \bmod \omega^{2}, \pi_{0}, \ldots, \pi_{k}$ gives

$$
T_{k+2, k+1}^{k+3}=0, X_{k+3, k}=-T_{k+2, k+1}, X_{k+2, k}=-T_{k+2, k+1}^{-1} .
$$

Upon successively evaluating $d \Phi \equiv 0 \bmod \pi_{0}, \ldots, \pi_{k}, \theta_{k+1}, \theta_{k+2}$ and $\bmod \pi_{0}, \ldots, \pi_{k}$, $\theta_{k+1}, \omega^{1}$ with these relations, we get

$$
Y_{k+2}=p_{k+3} T_{k+2, k+1}^{-1}, X_{k+3, k-1}=-T_{k+2, k+1}^{-1} .
$$

Now $, d \Phi \equiv 0 \bmod \omega^{2}, \pi_{0}, \ldots, \pi_{k-1}, \theta_{k+1}, \theta_{k+2}$ gives

$$
T_{k+2, k+1}^{-1}=0
$$

and we have

$$
d T_{k+2, k+1} \equiv T_{k+2, k+1}^{0} \theta_{0}+\ldots+T_{k+2, k+1}^{k+2} \theta_{k+2} \quad \bmod \alpha, \beta, \omega^{2} .
$$

Finally,

$$
d\left(d T_{k+2, k+1}\right) \equiv 0 \quad \bmod \quad \alpha, \beta, \omega^{2}, \theta_{0}, \ldots, \theta_{i}
$$

for $i=k+2, k+1, \ldots, 2$ implies $T_{k+2, k+1}^{i}=0$.

Since the equation $d \Phi=0$ is a linear differential system for the coefficients $A$, $B, C, T_{i+1, i}, X_{i+2, i}, X_{j+3, i}$, and $Y_{i}$, we have the following corollary.

Corollary 3.6. For a conservation law $\Phi \in \mathcal{H}_{k+3}$, all of $X_{i+2, i}, X_{j+3, i}$, and $Y_{i}$ can be expressed in terms of the principal coefficients $A, B, C,\left\{T_{i+1, i}\right\}_{i=0}^{k+1}$ and their successive covariant derivatives.

Thus, for each $k+3, k \geq-2$, there is a linear differential system

$$
D_{k+3}=D_{k+3}\left[T_{k+2, k+1}, T_{k+1, k}, \ldots, T_{1,0}, A, B, C\right]
$$

on $\mathcal{H}_{k+3}$ whose solutions correspond to the conservation laws in $\mathcal{H}_{k+3}$.

The theorem also suggests and justifies the following definition.

Definition 3.7. Let $\Phi \in \mathcal{H}_{\infty}$ be a conservation law. We define

$$
\text { weight } \Phi=|\Phi|=\max _{T_{i+1, i} \neq 0, i \geq 0} 2 i+1 \text {. }
$$


For a conservation law $\Phi \in \mathcal{H}_{1}$, we set $|\Phi|=-1$.

We also define

$$
\begin{aligned}
\mathrm{C}_{0} & =0, \\
\mathrm{C}_{k+3} & =\left\{\Phi \in \mathcal{H}_{k+3} \mid d \Phi=0\right\} \\
& =\{\text { conservation laws of weight at most } 2 k+3\}, \\
\mathcal{C}_{k+3} & =C_{k+3} / C_{k+2} \\
& =\{\text { conservation laws of weight } 2 k+3\} .
\end{aligned}
$$

\section{Corollary 3.8.}

$$
\mathcal{C}_{k+3}=\left\{\Phi \in C_{k+3} \mid T_{k+2, k+1} \neq 0\right\},
$$

and

$$
\begin{aligned}
\mathcal{C}_{\infty} & =\lim _{k \rightarrow \infty} \mathcal{C}_{k+3} \\
& =\bigcup_{k=-2}^{\infty} \mathcal{C}_{k+3} .
\end{aligned}
$$

The results in this section can be summarized as follows. Along with the prolongation tower $\mathrm{F}_{k+3}$,

$$
\mathrm{F}_{\infty} \rightarrow \mathrm{F}_{k+3} \rightarrow \mathrm{F}_{2}=\mathrm{F},
$$

we attach the subspaces $\mathcal{H}_{k+3}$,

$$
\bigwedge^{2} \mathcal{I}_{\infty} \supset \mathcal{H}_{\infty} \rightarrow \mathcal{H}_{k+3} \rightarrow \mathcal{H}_{2} \rightarrow \mathcal{H}_{1}
$$

and a sequence of linear differential systems $D_{k+3}$,

$$
D_{\infty} \rightarrow D_{k+3} \rightarrow D_{2} \rightarrow D_{1}
$$

whose solutions are the conservation laws of weight at most $2 k+3$. The sequence $\left\{D_{k+3}\right\}_{k=-2}^{\infty}$ enjoys the property

$$
D_{k+3}=\left.D_{\infty}\right|_{T_{i+1, i}=0} \text { for } i \geq k+2 .
$$

We finally mention that the weight of a conservation law roughly corresponds to the number of $x$ derivatives of $u$ involved in local coordinate expression, which agrees with the fact the orders of the conservation laws of the $K d V$ equation jump by two.

\section{EXAMPLES}

We compute the conservation laws of weight at most 3 for two classes of differential systems. A differential system will be called type $\left(n_{-1}, n_{1}, n_{3}\right)$ if it has $n_{i}$ conservation laws of weight $i$. 
4.1. Nonlinear differential systems with invariant $N=0$ that have at least one conservation law of weight $-1,1$, and 3 respectively. A complete classification can be given for this class of differential systems, where essentially the nonlinearity assumption allows us to close up the linear differential system $D_{3}$. First of all, a necessary condition to have a conservation law of weight -1 is $K=0$, and imposing the condition $N=0$ and (5) of Corollary 3.5, the structure equations in Proposition 1.1 become

$$
d\left(\begin{array}{c}
\omega^{2} \\
\theta_{0} \\
\omega^{1} \\
\theta_{1} \\
s_{2}
\end{array}\right)=\left(\begin{array}{c}
0 \\
-\theta_{1} \wedge \omega^{1} \\
0 \\
-s_{2} \wedge \omega^{1} \\
\left(G \theta_{1}+L \theta_{0}\right) \wedge \omega^{1}
\end{array}\right)
$$

on a section $\alpha=\beta=0$, which exists since $d \alpha=d \beta=0$. It is easily verified that these equations imply the following simple local normal form:

$$
\begin{aligned}
\omega^{2} & =d t, \omega^{1}=d x, \\
\theta_{0} & =d u-p d x, \\
\theta_{1} & =d p-q d x, \\
s_{2} & =d q+g(x, u, p) d x
\end{aligned}
$$

which corresponds to the evolutionary pde

$$
u_{t}=u_{x x x}+g(x, u, p) .
$$

Notice the admissible point transformations at this stage are, up to scaling by constants,

$$
\begin{aligned}
& \bar{x}=x+x_{0}, \\
& \bar{u}=u+\phi(x),
\end{aligned}
$$

where $x_{0}$ is a constant and $\phi(x)$ is an arbitrary function of $x$.

Following the general theory developed earlier, a conservation law $\Phi \in \mathcal{H}_{3}$ takes the form

$$
\begin{aligned}
\Phi & =A\left(\theta_{0} \wedge \omega^{1}+s_{2} \wedge \omega^{2}\right)-B \theta_{1} \wedge \omega^{2}+C \theta_{0} \wedge \omega^{2} \\
& +T \theta_{1} \wedge \theta_{0} \\
& +P\left(\theta_{2} \wedge \theta_{1}+s_{3} \wedge \pi_{0}\right),
\end{aligned}
$$

where

$$
\begin{aligned}
& \pi_{0}=\theta_{0} \quad-p_{3} \omega^{2}, \\
& \theta_{2}=s_{2}-p_{3} \omega^{1}, \\
& s_{3}=d p_{3} .
\end{aligned}
$$

A direct computation shows $d \Phi=0$ if

$$
\begin{aligned}
d P & \equiv 0 \quad \bmod \quad \omega^{2}, \\
T^{3} & =T^{2}=0, T^{-1}=A^{1}+g_{u} P, \\
A^{3} & =0, A^{2}=T, A^{-1}=B, \\
B^{3} & =0, B^{2}=P^{-2}-A^{1}, B^{-1}=C-g_{p} A-p_{3} P^{-2}, \\
C^{3} & =0, C^{2}=A^{0}, C^{1}=T^{-2}-B^{0}, C^{-1}=g_{u} A+A^{-2},
\end{aligned}
$$


where we express the covariant derivative of a coefficient $X$ by

$$
d X=X^{-1} \omega^{1}+X^{-2} \omega^{2}+X^{0} \theta_{0}+X^{1} \theta_{1}+X^{2} s_{2}+X^{3} s_{3} .
$$

Note by Theorem 3.4 ,

$$
\begin{aligned}
& \Phi \in \mathcal{H}_{2} \quad \text { if } \quad P=0 \\
& \Phi \in \mathcal{H}_{1} \quad \text { if } \quad P=T=0 .
\end{aligned}
$$

From now on, we give only the sketch of the procedure of overdetermined pde machinery that is applied to find the compatibility conditions to admit the desired conservation laws. When $X, Y, \ldots$ are the coefficients of the conservation laws and their covariant derivatives, the notation $\{X, Y, \ldots\}$ for simplicity would mean an expression that is linear in $X, Y, \ldots$ with the coefficients algebraic in $g(x, u, p)$ and its derivatives. For example, an expression like $g_{u}^{2} P+g_{p} A^{-2}$ will be denoted by $\left\{P, A^{-2}\right\}$. We also mention that, if there exists a compatibility condition of the form

$$
\{A, T, P\}=0 \text {, }
$$

all of the coefficients of $A, T$, and $P$ must be 0 if the differential system would admit each of the conservation laws of weight $-1,1$, and 3 .

1. $d(d(A)) \equiv 0 \bmod \omega^{2}, \theta_{0}, \theta_{1}$ gives $A^{1}=\left\{P, P^{-2}\right\}$.

$d(d(T)) \equiv 0 \bmod \omega^{2}, \theta_{0}, \theta_{1}$ gives $T^{1}=0$.

$d(d(T)) \equiv 0 \bmod \omega^{2}, \theta_{0}$ gives $T^{0}=\{P\}$.

$d(d(T)) \equiv 0 \bmod \omega^{1}, \omega^{2}$ gives $g_{p p u}=0$, which implies

$$
g(x, u, p)=h(x, u) p+a(x, u)+c(x, p)
$$

for some functions $h, a$, and $c$.

But, $d(d(T)) \equiv 0 \bmod \omega^{2}$ gives $a_{u u}=h_{x u}$, which suggests writing

$$
g(x, u, p)=f(x, u)_{x}+c(x, p) .
$$

2. $d(d(B)) \equiv 0 \bmod \omega^{2}, \theta_{0}, \theta_{1}$ gives $B^{1}=\left\{P, T, A^{0}\right\}$.

$d(d(A)) \equiv 0 \bmod \omega^{2}$ gives $A^{0-1}=\left\{B^{0}, T\right\}, A^{01}=\{P\}, A^{02}=\{P\}$.

$d(d(B)) \equiv 0 \bmod \omega^{2}, \theta_{0}$, gives $B^{0}=\left\{P, P^{-2}, T, T^{-2}, A\right\}$.

$d(d(B)) \wedge \omega^{1} \wedge \omega^{2}+\frac{1}{3} d(d(T)) \wedge \omega^{1} \wedge \theta_{0}=0$ gives $A^{00}=\left\{P, P^{-2}, T, A\right\}$.

$d\left(d\left(A^{0}\right)\right) \equiv 0 \bmod \omega^{1}, \omega^{2}, P$ gives $c_{p p p p}=0=c_{p p p x}$, which implies $c(x, p)$ is at most cubic in $p$.

We consider the case $c(x, p)$ is quadratic in $p$, for example, and write

$$
g(x, u, p)=f(x, u)_{x}+c_{2}(x) p^{2} / 2+c_{1}(x) p
$$

after adding some function of $x$ to $u$ if necessary.

3. $d\left(d\left(A^{0}\right)\right) \equiv 0 \bmod \omega^{2}, \theta_{1}, P, T$ gives $c_{2}(x)=0$.

$d\left(d\left(A^{0}\right)\right) \equiv 0 \bmod \omega^{1}, \omega^{2}$, gives $f_{\text {uuuu }}=0=f_{\text {uuux }}$, and $f(x, u)$ is at most cubic in $u$.

We again consider the case $f(x, u)$ is quadratic in $u$ and write

$$
g(x, u, p)=\left(f_{2}(x) u^{2} / 2+f_{1}(x) u+f_{0}(x)\right)_{x}+c_{1}(x) p,
$$

assuming $f_{2}(x) \neq 0$ for nonlinearity. Again by adding a suitable function of $x$ to $u$, we may write

$$
g(x, u, p)=\left(f_{2}(x) u^{2} / 2+f_{0}(x)\right)_{x}+c_{1}(x) p .
$$

4. $d\left(d\left(A^{0}\right)\right) \wedge \omega^{2}+\frac{1}{3} d(d(T)) \wedge \omega^{1}=0$ gives $P^{-2}=\{P\}, f_{2}^{\prime}=0$. 
Hence $f_{2}$ is a nonzero constant and after scaling, we may write

$$
\begin{gathered}
g(x, u, p)=\left(u^{2} / 2+f_{0}(x)\right)_{x}+c_{1}(x) p . \\
d(d(P))=0 \text { gives } c_{1}^{\prime \prime}=0, \text { and we put } c_{1}(x)=r_{1} x+r_{0} \text { with constants } r_{1}, \\
r_{0} . \\
d(d(B)) \wedge \omega^{2}+\frac{1}{3} d(d(T)) \wedge \theta_{0}=0 \text { gives } C^{0}=\left\{P, A, A^{0}\right\} . \\
d(d(C)) \wedge \omega^{2}+d(d(A)) \wedge \omega^{1}+\frac{2}{3} d(d(T)) \wedge \theta_{1}=0 \text { gives } \\
A^{0,-2}=\left\{P, T, T^{-2}, A^{0}, B\right\} . \\
d\left(d\left(A^{0}\right)\right) \wedge \omega^{1}-\frac{1}{3}\left(u+c_{1}(x)\right) d(d(T)) \wedge \omega^{1}=0 \text { gives } T^{-2}=\{P, T\} . \\
\text { Finally, } d(d(T))=0 \text { gives } f_{0}^{\prime \prime}=-2 r_{1}^{2} \text { and we put } \\
g(x, u, p)=\left(u^{2} / 2+\left(-r_{1}^{2} x^{2}+b_{1} x\right)\right)_{x}+\left(r_{1} x+r_{0}\right) p
\end{gathered}
$$

for some constant $b_{1}$.

5. $d\left(d\left(A^{0}\right)\right)=0$ gives $C=\left\{P, T, A, A^{0}, B\right\}$, and the exterior derivative of this relation gives $A^{-2}=\left\{P, T, A, A^{0}, B\right\}$. Now, by successively taking $d(d(A))$, $d(d(B))$, the remaining variables $B^{-2}$ and $C^{-2}$ are determined in terms of the free variables $P, T, A, B, A^{0}$, and the linear differential system for these free variables becomes completely integrable. Since

$$
\begin{array}{ll}
d P \equiv 0 & \bmod \quad P, \\
d T \equiv 0 & \bmod \quad P, T,
\end{array}
$$

this class of differential systems (equations) are type $(3,1,1)$.

Computations similar to the one demonstrated above determine the following list of (nonlinear) differential systems (equations) and their types.

$$
\begin{aligned}
& u_{t}=u_{x x x}+\left(\frac{1}{2} u^{2}-r_{2}^{2} x^{2}+r_{1} x\right)_{x}+r_{2} x p \quad(3,1,1), \\
& u_{t}=u_{x x x} \pm\left(\frac{1}{6} u^{3}+r_{1} x u+r_{0} u\right)_{x} \quad(2,2,1), \\
& u_{t}=u_{x x x}+\left(m^{\prime} \cos (u)+n^{\prime} \sin (u)\right)_{x}+\frac{1}{8} p^{3}+b p \\
& u_{t}=u_{x x x}+(m \cosh (u)+n \sinh (u))_{x}-\frac{1}{8} p^{3}+b p \\
& \left.u_{t}=u_{x x x} \pm(\exp (u))_{x}-\frac{1}{8} p^{3}+\left(r_{1} x+r_{0}\right) p+2 r_{1}, 1,2\right), \\
& u_{t}=u_{x x x} \pm \frac{1}{6} p^{3}+\left(r_{1} x+r_{0}\right) p+b
\end{aligned}
$$

where $r_{2}, r_{1}, r_{0}, b, m^{\prime}, n^{\prime}, m, n$ are constants such that $m^{2} \neq n^{2}$.

4.2. Flow of a curve in the plane by the derivative of its curvature with respect to the arc length. Consider the two-dimensional abelian group $R^{2}$ with a translation invariant Finsler structure, i.e., a translation invariant norm on the tangent vectors $\left[\mathrm{B}\right.$. It is known that there exists a coframe $\left\{\eta^{1}, \eta^{2}, \eta^{3}\right\}$ on the 
unit circle bundle $\mathcal{B} \rightarrow R^{2}$ with the structure equations

$$
\begin{aligned}
& d \eta^{1}=\eta^{3} \wedge \eta^{2}, \\
& d \eta^{2}=-\eta^{3} \wedge\left(\eta^{1}-I \eta^{2}\right), \\
& d \eta^{3}=0,
\end{aligned}
$$

where $I$ is the fundamental invariant satisfying

$$
d I \equiv 0 \quad \bmod \quad \eta^{3} .
$$

Here $\eta^{1}$ is the Hilbert form and $\eta^{3}$ is the canonical pseudo-connection form.

Given an immersed curve $\gamma$ in the plane, $\bar{\gamma}^{*}\left(\eta^{1}\right) \neq 0$, we define as usual the curvature of $\gamma$ by

$$
\bar{\gamma}^{*}\left(\eta^{3}\right)=k \bar{\gamma}^{*}\left(\eta^{1}\right)
$$

where $\bar{\gamma}$ is the canonical lift of $\gamma$ to $\mathcal{B}$. We also define $k_{1}$, the derivative of $k$ with respect to the arc length, by

$$
d k=k_{1} \bar{\gamma}^{*}\left(\eta^{1}\right) .
$$

The $k_{1}$-flow we are interested in is the following. Given $\gamma \subset R^{2}$, first lift it to $\bar{\gamma} \subset \mathcal{B}$. Then flow $\bar{\gamma}$ along the Finsler normal, or the vector dual to $\eta^{2}$, in the amount of $k_{1}$. The image of this flow under the projection $\mathcal{B} \rightarrow R^{2}$ will be called $k_{1}$-flow of $\gamma$.

$k_{1}$-flow can be expressed in terms of a differential system on a five manifold $\mathrm{M}^{5}=\{t\} \times\{k\} \times \mathcal{B}$ as follows:

$$
\mathcal{I}=\left\{\theta_{0} \wedge \omega^{1}+\sigma_{2} \wedge \omega^{2}, \theta_{1} \wedge \omega^{2}, \theta_{0} \wedge \omega^{2}\right\} \cup\left\{\theta_{1} \wedge \theta_{0}\right\},
$$

where

$$
\begin{aligned}
\omega^{2} & =d t, \\
\theta_{0} & =\eta^{2}, \\
\omega^{1} & =\eta^{1}-I \eta^{2}, \\
\theta_{1} & =\left(\eta^{3}-k \eta^{1}\right)-k I \eta^{2}, \\
\sigma_{2} & =d k,
\end{aligned}
$$

with the independence condition $\omega^{1} \wedge \omega^{2} \neq 0$. It is clear an integral manifold of $\mathcal{I}$ on which $\omega^{1} \wedge \omega^{2} \neq 0$ is locally the graph over the canonical lift of a $k_{1}$ flow.

Computation as in example 4.1, i.e., repeated application of the identity $d^{2}=0$, yields the following results.

(a) case $I=0$.

The Finsler structure in this case is the flat Riemannian metric on $R^{2}$. The differential system is type $(4,1,1)$. If the initial curve $\gamma$ is simply closed, the first five conservation laws represent the preservation under the flow of

$$
\int_{D} c_{1}+c_{2} x+c_{3} y+c_{4}\left(x^{2}+y^{2}\right) d x \wedge d y,
$$

and the length of the curve, where $(x, y)$ are the orthonormal coordinates of the plane, $D$ is the region enclosed by the curve, and $c_{i}$ are constants. We also mention $\gamma$ admits a self similar $k_{1}$-flow if

$$
k_{1}^{2}+\frac{1}{4} k^{4}+a_{2} k^{2}+a_{1} k+a_{0}=0
$$


for some constants $a_{i}$. It is interesting to note that upon imposing $a_{2}=a_{1}=0$, the above ordinary differential equation for $k$ becomes the Euler-Lagrange equation for the functional

$$
\int_{\gamma} \frac{1}{2} k^{2}
$$

In ChT, it is shown the curvature of the $k_{1}$-flow satisfies the $m K d V$ equation under a suitable local coordinates system.

In case $I \neq 0$, the differential system does not have any conservation laws of weight -1 . Below are the Finsler structures whose $k_{1}$-flow have at least one conservation law of weight 1 and 3 .

(b) case $I \neq 0, d I=\left(-\frac{3}{2}+\frac{1}{3} I^{2}\right) \eta^{3}$.

It is type $(0,3,2)$, and also has one conservation law of weight 5 . There does not exist a Finsler structure realizing this structure equation $\mathrm{B}$.

(c) case $I \neq 0, d I=I_{3} \eta^{3} \neq\left(-\frac{3}{2}+\frac{1}{3} I^{2}\right) \eta^{3}, d I_{3}=\left(-I+\frac{2}{9} I^{3}\right) \eta^{3}$.

It is type $(0,3,1)$, and also has one conservation law of weight 5 .

Remark. Each of the differential systems presented in the list (6) of example 4.1 and (a), (b), (c) of example 4.2 also has exactly one conservation law of weight 5 . After computing the conservation laws of these and other similar examples, it is tempting to conjecture the following.

Consider a differential system that locally corresponds to a nonlinearizable (nonlinear) evolutionary pde of the form

$$
u_{t}=f\left(x, u, u_{x}\right) u_{x x x}+g\left(x, u, u_{x}, u_{x x}\right) .
$$

If the differential system has conservation laws of weight $-1,1$, and 3 , then it has a conservation law of weight $2 k+3$ for each $k \geq 1$. In general, if the differential system has three conservation laws of distinct weights greater than or equal to 1 , then it has an infinite sequence of conservation laws of distinct weights.

\section{REFERENCES}

[B] R. Bryant, Finsler surfaces with prescribed curvature conditions, preprint (1995).

[BG1] R. Bryant and P. A. Griffiths, Characteristic cohomology of differential systems I, J. Amer. Math. Soc. 8 (1995), 507-596. MR 96c:58183

[BG2] _ _ Characteristic cohomology of differential systems II, Duke Math. J. 78 (1995), 531-676. MR 96d:58158

[BG3] __, Reduction for constrained variational problems and $\int \frac{1}{2} \kappa^{2} d s$, Amer. J. Math. 108 (1986), 525-570. MR 88a:58044

[ChT] S. S. Chern and K. Tenenblat, Foliations on a surface of constant curvature and the modified Korteweg-De Vries equations, J. Diff. Geom. 16 (1981), 347-349. MR 83i:58004

[F] K. Foltinek, Quasilinear third-order scalar evolution equations and their conservation laws, Thesis, Duke university (1996).

[K] O. V. Kaptsov, Classification of evolution equations by conservation laws, Functional Analysis and its Appl. 16 no.1 (1982), 59-61. MR 83h:58052

[M] A. V. Mikhailov, A. B. Shabat, and V. V. Sokolov, The symmetry approach to classification of integrable equations, What is integrability?, Springer Series in Nonlinear Dynamics, Springer-Verlag, Berlin (1991). MR 93b:58070

Department of Mathematics, Postech, Pohang, Korea 790-784

E-mail address: wang@postech.ac.kr 